\title{
Kurtosis Based Empirical Mode Decomposition for Rolling Bearing Fault Detection
}

\author{
Hui $\mathrm{Li}^{*}$ and Xuhan Liu \\ School of Mechanical Engineering, Tianjin University of Technology and Education, Tianjin, China
}

\begin{abstract}
A bearing fault diagnosis approach based on spectral kurtosis and empirical mode decomposition (EMD) is proposed. EMD is a signal decomposition technique, which can adaptively separate a number of intrinsic mode functions (IMFs) from the vibration signal according to the architectural characteristics of the data. The spectral kurtosis parameter takes as signal impulsive indicator. Firstly, EMD is utilized to process the sampling vibration signal. And then spectral kurtosis is calculated to select the optimal intrinsic mode functions, so as to suppress the noise and highlight the transient impact feature. Finally, the envelope spectrum is computed and the fault characteristic is recognized. The experimental results show that the proposed approach can identify bearing defects effectively and provide a reliable method for gearbox fault monitoring and diagnosis.
\end{abstract}

\section{Introduction}

Bearings are important mechanical components in various mechanical equipments, such as automobile, metallurgical equipment, etc. The unexpected failure of bearings will affect the normal operation of the equipment, and even cause significant economic losses. Therefore, bearing fault detection and diagnosis has been a hot topic for scholars. Among many methods of bearing fault feature extraction, the approach based on vibration signal analysis technique is widely utilized because of its high reliability. For example, fast Fourier transform (FFT), power spectrum estimation, envelope spectrum and cepstrum analysis technique have achieved good results in bearing fault detection and diagnosis $[1,2]$. However, All of these signal processing technique are all in view of the hypothesis of linearity and stationarity of vibration signals. But the vibration signal of rolling bearing defect has the characteristics of nonlinear and non-stationary, and the vibration signal of bearing fault has the characteristics of transient impact in essence. Therefore, the rolling bearing system defect recognition and diagnosis need to study new vibration analysis technology.

This paper put forwards an approach of bearing fault monitoring and recognition based on EMD and spectrum kurtosis based envelope spectrum. EMD is a new nonstationary, nonlinear adaptive signal decomposition method proposed by Huang [3]. EMD can adaptively separate multi component signals into a number of intrinsic mode functions (IMF). Then the spectrum kurtosis is utilized to select the optimal parameters of band-pass filter, which can effectively suppress the noise in the signal and highlight the transient impact characteristics of the signal. Finally, the envelope spectrum of the selected band-pass signal is analyzed, and the bearing fault is identified and diagnosed according to the spectrum characteristics of the envelope spectrum. The envelope spectrum method based on spectral kurtosis can effectively extract the feature frequencies related to bearing defects. The basic principle of the approach is presented briefly, and the method is applied to bearing defect monitoring and recognition. Experimental results show that this approach can effectively monitor and identify rolling bearing faults.

\section{The theory of kurtosis based EMD}

In 1998, Huang et al [3,4] defined the IMFs and put forward the EMD technique. EMD is an effective technique for nonlinear and non-stationary signal processing.

\subsection{Empirical mode decomposition}

EMD method is put forward based on the hypothesis of simple morphological diversity, that is, any vibration signal is composed of function superposition with various of simple natural vibration modes. The innate character of this technique is to use the characteristic time scale of the vibration signal to identify the intrinsic vibration mode of the signal, and then the signal is adaptively decomposed into the sum of several IMFs. The characteristic time proportion can be defined by the time difference between two consecutive extremes.

In order to pick up IMF adaptively from a vibration signal, the signal sifting process is given as bellow. Firstly, all local extrema of vibration signal, including local maximum and local minimum, are determined, and

\footnotetext{
* Corresponding author: huili68@163.com
} 
then all local maxima are fitted together by cubic spline as upper envelope. Then, the local minima are fitted together to generate the lower envelope of the vibration signal. The upper and lower envelope lines of vibration signals shall contain all points between them. Their average value is defined as $f_{1}(t)$, the difference between the data and $f_{1}(t)$ is defined as $d_{1}(t)$, i.e.:

$$
x(t)-f_{1}(t)=d_{1}(t)
$$

In an ideal situation, $d_{1}(t)$ should be an intrinsic mode function, because the methods and steps of sifting $d_{1}(t)$ represented above should force the results to meet all conditions of the IMF by constructing $d_{1}(t)$. To check whether $\mathrm{h}$ is an IMF, $d_{1}(t)$ only needs to meet the following conditions: (i) $d_{1}(t)$ should not be affected by riding waves, that is, the first component should not be under-shoot or over-shoot displayed on the data, and local extremum should be generated without zero crossing. (ii) The displayed upper and lower envelope lines are symmetrical with regard to zero. (iii) Clearly, the number of zero crossing points and extreme points should be the same in these two functions.

Such a data filtering process may be repeated many times to ensure that the extracted signal is an IMF. In the subsequent sifting, $d_{1}(t)$ is treated as the data to be processed, as follows:

$$
d_{1}(t)-f_{11}(t)=d_{11}(t)
$$

Where $f_{11}(t)$ is the average value of the upper envelope and the lower envelope of $d_{1}(t)$.

The above mentioned process should be redone $k$ times and $d_{1 k}(t)$ is given as follow

$$
d_{1(k-1)}(t)-f_{1 k}(t)=d_{1 k}(t)
$$

After each filtering step, it must be checked whether the number of zero crossing points and the number of extreme values are exactly the same.

The final shifting result is the first IMF, and then it is appointed as $c_{1}(t)=d_{1 k}(t)$. The first IMF element from the data should embody the highest vibration frequencies searched in the original signal.

Then subtract the first IMF from the original signal, and the difference, is called a residue $r_{1}(t)$ which is formulated as follow:

$$
x(t)-c_{1}(t)=r_{1}(t)
$$

The residue $r_{1}(t)$ is taken as if it was the original data and we apply to it again the sifting process. The data shifting process of finding more intrinsic modes $c_{i}(t)$ will be repeated many times to find all the intrinsic mode functions in the signal until the last one is found. The final residue will be a monotonic function or a constant. In general, The residue will be the general trend of data.

$$
x(t)=\sum_{i=1}^{n} c_{i}(t)+r_{n}(t)
$$

After the above steps, the original data is effectively separated into $n$ number of intrinsic mode functions and a residual, which can be an average trend or a constant [5].

\subsection{Spectral kurtosis}

Spectral kurtosis is the fourth-order statistic of the signal, which is mainly used to calculate the spectral kurtosis in the frequency spectrum map, so as to highlight the transient impulsive characteristics of the primitive vibration data. This approach conquers the shortcoming that the Fourier transform can't monitor and identify the transient impact feature of original sampled vibration data.

Spectral kurtosis represents the peak of probability density function, which can be formulated as follow

$$
K_{x}(f)=\frac{S_{4 x}(f)}{S_{2 x}^{2}(f)}-2
$$

Where $S_{4 x}(f)$ is the fourth order cumulant of the spectral density of the original vibration data $x(t)$ and $S_{2 x}(f)$ is the second order cumulant.

When the sampled vibration data $x(t)$ contains noise, the spectral kurtosis is given as follows:

$$
K_{x}(f)=\frac{K_{x}(f)}{|1+\rho(f)|^{2}}
$$

Where $K_{x}(f)$ is the spectral kurtosis of original vibration data $x(t)$ without noise and $\rho(f)$ is the count backwards of signal to noise ratio.

The magnitude of the kurtosis reflects the severity of the transient shock phenomenon in the original vibration data. It is related to the resonance frequency band of the fault vibration signal of the selected bearing. The premise of accurately extracting and identifying bearing fault features is to find the frequency band center and its bandwidth where the peak value of spectrum lies.

\section{Bearing damage detection based on kurtosis based EMD}

The experimental ball bearing is only utilized to research one kind of localized surface defect: bearing outer or inner raceway defect. The tested rolling bearing is used discharge device to machine a slot on the inner or outer race. Then the localized fault is a seed formed on the outer or inner raceway. The defect was $1 \mathrm{~mm}$ deep and $1.5 \mathrm{~mm}$ wide. The gearbox is driven by three-phase alternating current motor. The input spindle speed is $1500 \mathrm{r} / \mathrm{min}$, that is to say, $f_{r}$ is $25 \mathrm{~Hz}$. The bearing 
model is deep groove ball bearing 6208. The number of balls is 10 and the contact angle $\alpha=0^{\circ}$. The bearing pitch diameter $\mathrm{D}$ is $97.5 \mathrm{~mm}$ and the ball diameter $\mathrm{d}$ is $55 / 3 \mathrm{~mm}$. The rolling bearing defect characteristic frequencies of inner race is $148.5 \mathrm{~Hz}$ [6].

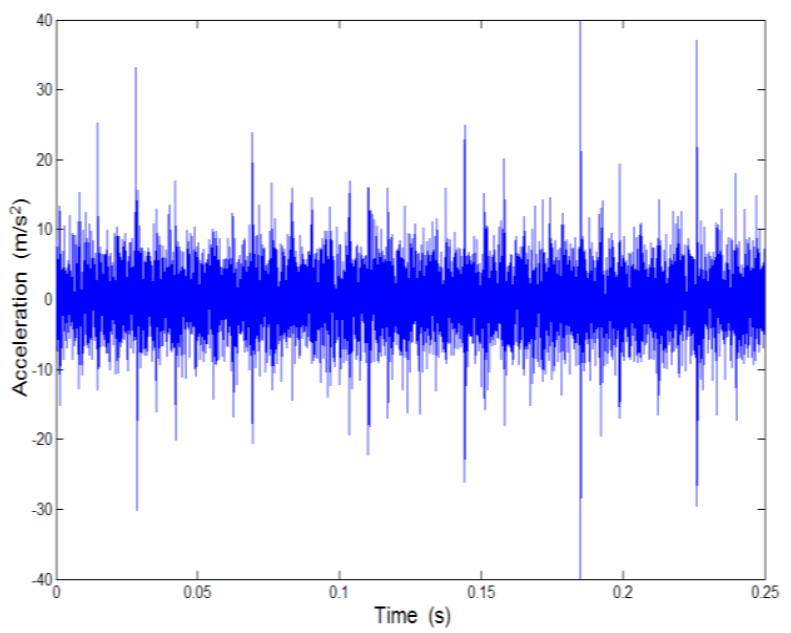

Fig. 1. Vibration data with inner defect.

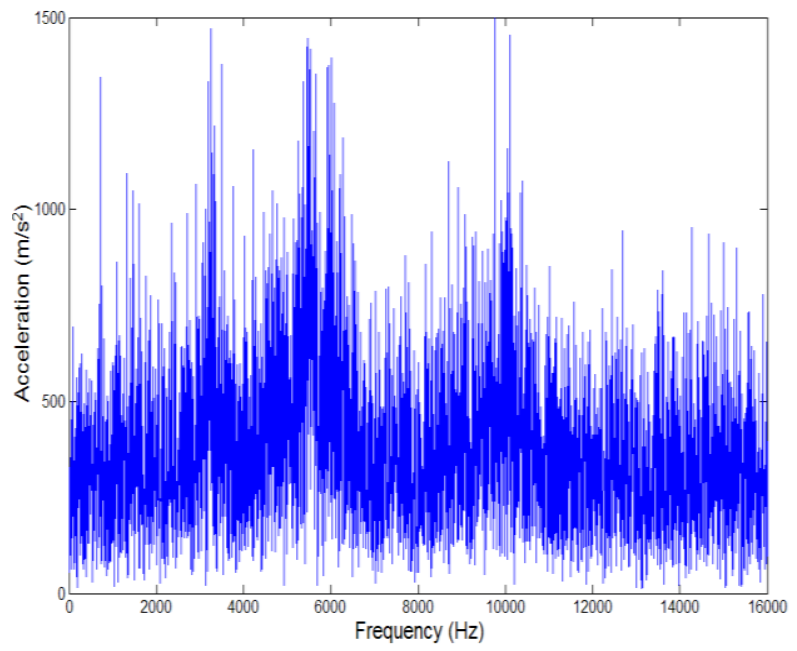

Fig.2. FFT with inner defect.

The sampled bearing inner race defect vibration data is displayed in Fig.1 and the FFT is showed Fig.2. In Fig. 2 , there is no significant spectral peak near the rolling bearing defect characteristic frequency $f_{\text {inner }}$ and its higher harmonics components. Therefore, the classical Fourier analysis can not accurately extract the transient impact characteristics of bearing fault in noise environment, so it is difficult to effectively diagnose bearing localized fault.

The EMD algorithm is utilized for the vibration data of Fig.1. Fig. 3 gives the empirical mode decomposition of vibration data with inner race defect, which has eleven intrinsic mode functions. There are eleven intrinsic mode functions: $c_{1}-c_{13}$, which selected from empirical mode decomposition. The IMFs represent the intrinsic modes of various frequency components actuated by bearing inner race fault, $c_{13}$ is the residue, respectively. Among them, $c_{1}$ has the highest frequency of the vibration data, $c_{2}$ represents the next higher frequency component, and others. These empirical modal functions clearly describe the transient vibration feature caused by bearing inner race fault. It can be easily proved from Fig. 3 that EMD is very effective in the decomposition of vibration signals.

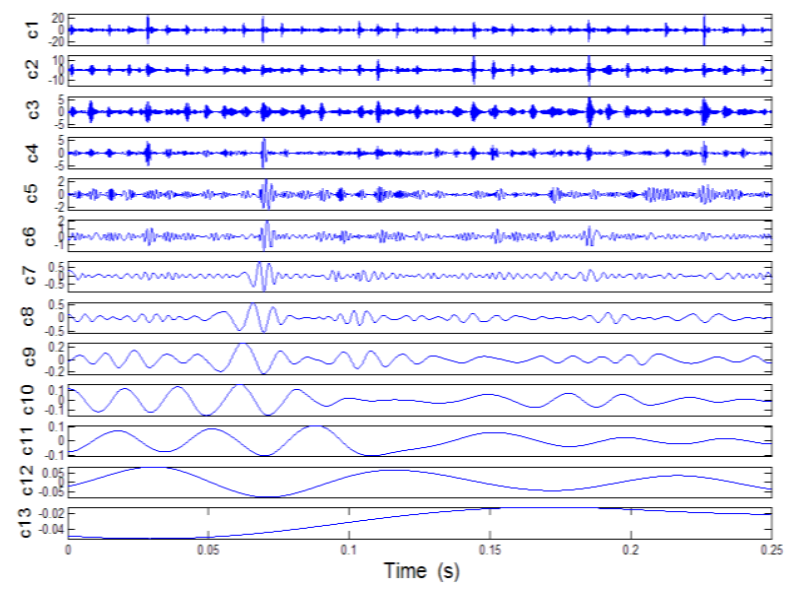

Fig. 3. IMFs of the data with inner defect.

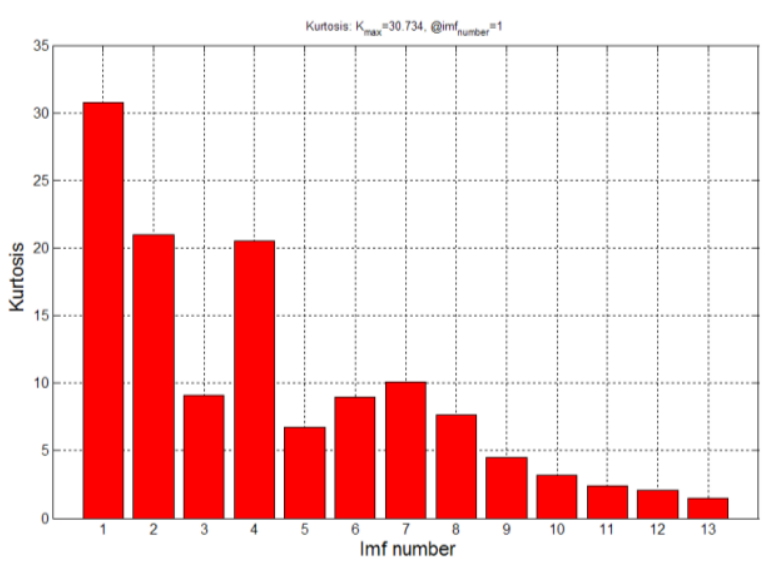

Fig. 4. Spectral kurtosis.
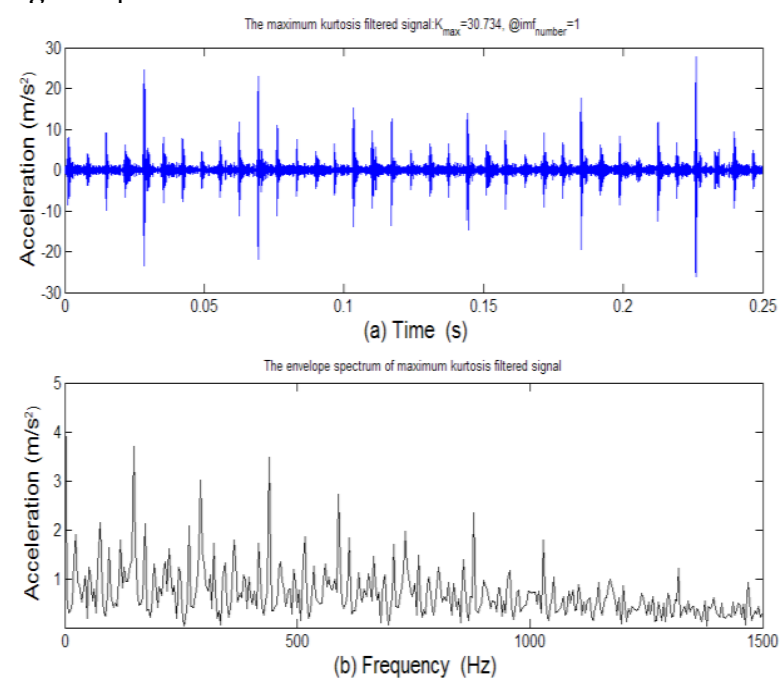

Fig. 5. The optimal kurtosis filtered data and its envelope spectrum.

In order to prove the effectiveness of the proposed method over the traditional envelope spectrum, Fig.4 
shows the kurtosis of each IMF and the maximum kurtosis is in $c_{1}$. Fig.5 shows the optimal kurtosis filtered signal $c_{1}$ and its envelope spectrum. In Fig. 5, one can clearly see the transient impact characteristics of the vibration data when the bearing inner race has defect. The fault of the bearing inner race leads to the sudden increase of vibration energy. For the faulty bearings, the transient impact feature caused by the interaction of roller defects can be clearly seen. In addition, these transient impacts reveal the failure mode of the bearing, corresponding to the bearing inner race characteristic fault frequency of $148.5 \mathrm{~Hz}$, which is caused by the structural defects of the inner race. This kind of repetition frequency reflects the deterioration of the inner raceway health when the bearing fault propagates through the bearing inner race.

\section{Conclusions}

In order to effectively extract the transient impact features of noisy vibration signals, an empirical mode decomposition method based on spectral kurtosis is proposed. This method has obvious advantages in extracting transient impact signal, which is often an important index of early defects of bearing system. Firstly, the vibration data is adaptively decomposed into several intrinsic modal functions by EMD, then the spectral kurtosis of each empirical modal function is calculated, and finally the envelope spectrum of the intrinsic mode function with the largest spectral kurtosis is calculated. The experimental results show that the
EMD based on spectral kurtosis can effectively extract bearing faults.

\section{Acknowledgment}

This research is a part of the research that is sponsored by the scientific research fund of Tianjin University of Technology and Education (No. KYQD202003). At the same time, I am very glad to express my sincere thanks to the anonymous reviewers.

\section{References}

1. R.Rubini, U.Meneghetti, Mechanical Systems and Signal Processing 15, 287-302 (2001)

2. D. Abboud, J. Antoni, S. Sieg-Zieba, et al, Mechanical Systems and Signal Processing 84, 200226 (2017)

3. N.E Huang, Z. Shen and S.R.Long et al, Proceeding of Royal Society London. Series A 454, 903-995 (1998)

4. N.E.Huang, Z.Shen and S.R.Long, Annual Review of Fluid Mechanics 31, 417-457 (1999)

5. H. Li, Y. Zhang, H. Zheng, Journal of Mechanical Science and Technology 20, 1781-1789 (2006).

6. H. Li, Y. Zhang, H. Zheng, Journal of Mechanical Science and Technology 23, 291-301 (2009) 